Relations industrielles

Industrial Relations

\title{
Les livres
}

\section{Les tribunaux du travail}

\section{Denys Dion}

Volume 5, numéro 8, mai 1950

URI : https://id.erudit.org/iderudit/1023368ar

DOI : https://doi.org/10.7202/1023368ar

Aller au sommaire du numéro

Éditeur(s)

Département des relations industrielles de l’Université Laval

ISSN

0034-379X (imprimé)

1703-8138 (numérique)

Découvrir la revue

Citer ce document

Dion, D. (1950). Les livres : les tribunaux du travail. Relations industrielles /

Industrial Relations, 5(8), 77-78. https://doi.org/10.7202/1023368ar

Tous droits réservés @ Département des relations industrielles de l’Université Laval, 1950
Ce document est protégé par la loi sur le droit d'auteur. L'utilisation des services d'Érudit (y compris la reproduction) est assujettie à sa politique d'utilisation que vous pouvez consulter en ligne.

https://apropos.erudit.org/fr/usagers/politique-dutilisation/ 


\section{C.-ERREURS}

Tout calcul de productivité doit être accompagné d'une évaluation de l'exactitude des résultats. Sans calcul d'erreur, les résultats d'une mesure de productivité n'ont aucune valeur scientifique et il est difficile de les prendre comme base d'étude.

Les calculs de productivité révèlent souvent des erreurs de l'ordre de 10 à 20\%. Dans des cas simples et bien délimités, les résultats peuvent être exacts à une ou deux unités pour cent près, mais dès que les mesures atteignent un domaine plus large, ou s'étendent dans le temps, une telle exactitude devient souvent impossible. D'ailleurs, une approximation de l'ordre de 10 à $20 \%$, dont il ne saurait être question dans un calcul de prix de revient, n'a qu'une importance relative dans un calcul de productivité.
Le plus souvent, en effet, les variations de productivité ont assez d'ampleur pour qu'une erreur de $20 \%$ devienne négligeable. (Il est fréquent d'enregistrer des productivités qui varient de 1 à 5 ou même plus). Enfin, le but des mesures est beaucoup plus d'obtenir des indices de comparaison que des valeurs absolues; dès lors une approximation, même de $20 \%$ près, est fort instructive du moment qu'elle met en évidence une tendance majeure ou des disparités beaucoup plus fortes. Il faut remarquer d'ailleurs, que l'emploi de méthodes de calcul précises et généralement adoptées doit réduire l'ordre de grandeur de ces erreurs à $10 \%$ au maximum.

Les calculs d'erreurs en productivité sont conduits selon les règles classiques en arithmétique. Il suffit de calculer l'erreur sur chacun des termes entrant dans le calcul, l'erreur sur le résultat en découle mathématiquement.

\title{
LES LIVRES
}

\section{LES TRIBUNAUX DU TRAVAIL}

\author{
DENYS DiON
}

Le Service extérieur d'éducation sociale de l'Université Laval vient de publier une plaquette pleine d'intérêt. C'est la première d'une série portant sur les relations du travail. Elle a pour titre « Les tribunaux du Travail » et pour auteur, M. Benoît Yaccarini, maître en sciences sociales et spécialiste en relations industrielles. C'est la condensation de la thèse que ce dernier a présentée à la Faculté des sciences sociales de l'Université Laval de Québec pour l'obtention de sa maîtrise. Cette étude avait été antérieurement présentée dans le numéro d'octobrenovembre 1949 de la Revue du Barreau.

Dans son introduction, l'auteur trace sommairement l'évolution de la législation sociale et il tente de souligner l'opposition qui existe de plus en plus marquée entre le droit traditionnel du Code civil, individualiste et libéral, et le développement d'un droit social, plus large et plus compréhensif.

Partant de ce point, l'auteur, dans la première partie de son travail, veut montrer ela nécessité qu'il y a aujourd'hui d'instituer des tribunaux du Travail en général et quel est le mécanisme de leur fonctionnement ». Puis, dans une seconde partie, il tentera d'appliquer à la province de Québec les principes généraux des tribunaux du Travail.

L'auteur divise la première partie de son ouvrage en cinq chapitres. Dans le premier chapitre, il expose le but des tribunaux du Travail qui est avant tout d'apporter une procédure appropriée aux conflits industriels, c'est-àdire une procédure moins coûteuse, moins formaliste, plus expéditive. Ces tribunaux ont encore pour but la nomination de juges bien au courant des habitudes développées dans les milieux de travailleurs », et des questions de fait que seuls les hommes de métier sont à même de connaître à fond, enfin des juges bien pénétrés de l'esprit et du sens de la législation du travail.

Dans son deuxième chapitre, l'auteur fait l'historique des tribunaux du Travail. Les juridictions spéciales du Travail existent, selon l'auteur, dans au moins trente pays. Ces tribunaux, presque tous semblables dans leur esprit, diffèrent sensiblement par leurs techniques. Le caractère de ces tribunaux dépend de l'évolution sociale et économique des pays qui les ont institués. Au début, ces tribunaux ne connaissaient que des conflits individuels, puis peu à peu ils en sont venus à connaître des conflits collectifs. L'auteur traite alors des organismes semi-administratifs et semi-judiciaires qu'on trouve aux Etats-Unis, au Canada et dans diverses de nos provinces, établis dans le but de régler certains conflits de travail. L'auteur fait remarquer qu'il «ne faut pas confondre les tribunaux du Travail, dont le rôle est de dire le droit (individuel ou collectif), avec les Services ou Conseils de conciliation et d'arbitrage, dont le rôle principal est de créer de nouveaux droits 》.

L'auteur consacre le chapitre suivant à la compétence des tribunaux du Travail. En d'autres termes, quel est le champ d'application ouvert à un tel tribunal? Il y a des tribunaux dont les sentences obligent et d'autres dont les sentences ne sont pas exécutoires. Et alors l'attribution des compétences sera motivée par la distinction entre conflits individuels et conflits collectifs, conflits de droit (individuels et collectifs) et conflits d'intérêts.

L'auteur s'applique à bien définir ces différents concepts qui sont à la base de la compétence des tribunaux du Travail. Cependant il insiste avec beaucoup de raison, semble-t-il, sur le fait que l'on devrait surtout baser l'attribution des compétences sur la distinction entre conflits de droit et conflits d'intérêts. La solution des conflits de droit devrait être confiée aux tribunaux du Travail alors que les organismes de conciliation et d'arbitrage se chargeraient des conflits d'intérêts.

Puis, au quatrième chapitre, l'auteur expose la procédure devant les tribunaux du Travail. Comme il le dit si bien, « cette procédure doit être simple, dégagée de tout formalisme, rapide et peu dispendieuse \$. En général, dit-il, les parties comparaissent en personne devant de tels tribunaux. Mais s'il s'agit d'une personne morale, il faut nécessairement qu'elle soit représentée par un mandataire. Or ce mandataire doit-il nécessairement être un homme de loi, un avocat? D'apprès M. Yaccarini, dans la plupart des pays où l'on a institué les tribunaux du Travail, 
on restreint ou même on empêche la représentation des parties par les avocats. L'une des principales raisons d'agir ainsi semble être le souci de rendre les procédures moins coûteuses. Cependant, le recours à l'homme de loi peut devenir presque nécessaire surtout dans les conflits de droit. Il arrive alors que certains gouvernements prévoient un tarif spécial ou encore l'assistance judiciaire ou bien un service gouvernemental d'aide aux parties économiquement faibles.

Il y a cependant une difficulté assez importante au sujet de l'exécution des jugements rendus par les tribunaux du Travail. Cela est relativement facile pour les conflits individuels, mais il n'en va pas de même pour les conflits collectifs. La difficulté se fait surtout sentir dans les pays à régime économique libéral. Elle n'est pas encore résolue. Pour finir sa première partie, l'auteur parle des avantages des tribunaux du Travail.

Dans la seconde partie de son ouvrage, l'auteur tente d'appliquer à la province de Québec les principes généraux des tribunaux du Travail étudiés dans la première partie.

Il débute en se demandant s'il est opportun d'instituer de tels tribunaux dans le Québec. A quoi il répond que \&l'établissement de tribunaux du Travail dans la province de Québec est devenu nécessaire et même urgent. Les voix les plus autorisées en la matière y sont favorables. Ces tribunaux réaliseraient une meilleure compréhension des problèmes du travail et contribueraient grandement à l'amélioration des relations industrielles. Leur procédure abrégée et simple, leur composition représentative des intérêts en conflit et enfin la modicité des frais imposés aux parties, inspireraient confiance à la classe laborieuse et sont autant de raisons légitimant leur création $\gg$.

Cependant, il se présente ici une difficulté d'ordre constitutionnel et politique. L'auteur a vite fait d'en disposer en démontrant que « la province de Québec a le droit d'instituer des tribunaux du Travail au moment qu'elle jugera le plus opportun. Rien dans la constitution de 1867, ajoute-t-il, ne permet au gouvernement fédéral de s'y opposer. » Mais l'auteur voit une autre difficulté en raison de l'article 98 de l'A.A.B.N., stipulant que * les juges des tribunaux du Québec seront choisis parmi les membres du Barreau de cette province ».

Or il ressort de l'étude précédente que « pour que les tribunaux du Travail atteignent leur fin, il est à conseiller qu'ils soient composés d'un nombre égal d'assesseurs représentant les employeurs et les salariés, auxquels on adjoint comme président un homme de loi, versé dans le droit ouvrier, juge de carrière ou avocat. C'est ainsi qu'on éluderait la difficulté, l'assesseur n'étant pas exactement un juge, son rôle étant surtout d'assister le juge, de le conseiller sur les questions de fait qui échappent à sa connaissance, délibérant tout de même avec le juge et prenant part effective au jugement. »

A ce point de son travail, l'auteur étudie la compétence des tribunaux du Travail. D'abord, compétence en raison de la nature du conflit. Pour lui, \& la solution idéale serait certainement celle qui donnerait compétence au tribunal du Travail de connaître toute espèce de conflits naissant des relations industrielles ».

Malheureusement une telle solution s'avère impossible ici, parce que les esprits, tant dans les groupes ouvriers que patronaux et gouvernementaux, n'y sont pas prêts. De plus, « il ne faut pas oublier, dit l'auteur, que, donner à un organisme quelconque, serait-ce un tribunal du Tra- vail, le pouvoir de réglementer définitivement et obligatoirement les conditions de travail équivaudrait en fait à une véritable réforme des structures économiques. Les salaires au Canada, d'après les statistiques, représentent plus de la moitié du revenu national; les réglementations par voie d'autorité aboutiraient, somme toute, à un début d'économie dirigée. » Il faudrait donc, conclut l'auteur, « procéder avec beaucoup de circonspection et par étapes ». Il faudrait d'abord étendre leur compétence aux conflits de droit d'une façon exclusive et très large. Ensuite il faudrait leur donner un pouvoir de sanction très étendu. Quant à la compétence territoriale, l'auteur suggère, avec raison, qu'il serait bon d'attribuer compétence au tribunal de la localité où le conflit est né ou à celui où le travail qui a donné naissance au conflit est effectué. Pour ce qui est de la composition du tribunal, l'idéal serait d'employer le système pluraliste où, à côté de l'élément professionnel patronal et ouvrier, serait compris l'élément judiciaire. Enfin, l'auteur parle de la procédure qui doit être, dit-il, expéditive et très peu onéreuse. Il ajoute que dans leurs sentences les magistrats devraient se laisser guider par des motifs d'équité autant que de droit.

Voilà, résumé à grands traits, l'étude de $\mathrm{M}$. Yaccarini sur les tribunaux du Travail. Que faut-il en penser? Il n'y a aucun doute possible que c'est un travail de grande importance et de grande actualité. L'auteur y a certainement apporté un grand effort de recherches et d'adaptation. Le sujet avait déjà été abordé avec maîtrise par des gens de haute capacité. M. Yaccarini a le mérite et l'originalité de synthétiser les données actuelles sur cette question et surtout de tenter une formule d'adaptation pour notre province. Sans endosser complètement toutes ses conclusions, on ne peut s'empêcher de lui rendre le témoignage d'avoir concouru d'une façon pratique à la solution d'un problème des relations du travail.

Je crois qu'il est bon de nuancer la formule d'application des tribunaux du Travail afin d'arriver à une meilleure adaptation de ces tribunaux à notre système de liberté. Il semble que, pour le moment, on devrait se contenter de créer des tribunaux du Travail dont la compétence s'étendrait seulement mais exclusivement aux conflits de droit.

L'auteur préconise l'exclusion des hommes de loi de l'immense champ du droit du Travail. Je ne crois pas que l'on puisse concourir dans son opinion. Son argumentation à ce sujet semble amener une certaine contradiction. L'auteur voudrait, en effet, que le président du tribunal du Travail soit un juge ou un avocat bien imprégné du «droit social 》 et versé dans les questions industrielles. Or je crois qu'il est bien difficile de devenir bien imprégné de «droit social » et versé dans les questions industrielles, à moins d'avoir une bonne expérience pratique en ce domaine. C'est pourquoi il semble préférable qu'on laisse aux avocats, surtout à ceux qui ont les qualifications spéciales, accès à ce champ des relations du travail. Cela n'exclut pas une certaine réglementation qui pourrait peut-être venir à la fois des corps professionnels et de l'Etat. Cette réglementation pourrait porter, entre autres choses, sur les tarifs.

Mais de toute manière, je ne crois pas qu'il soit désirable, dans notre régime, d'exclure les hommes de loi.

Ces réserves faites, je dois dire que le travail de $\mathbf{M}$. Yaccarini est digne d'éloges. 